\title{
Technique of robotic esophagectomy
}

\author{
Tadeusz D. Witek, John J. Brady, Inderpal S. Sarkaria \\ University of Pittsburgh Medical Center, University of Pittsburgh School of Medicine, Pittsburgh, PA, USA \\ Correspondence to: Inderpal S. Sarkaria. Shadyside Medical Building, 5200 Centre Ave, Suite 715, Pittsburgh, PA 15232, USA. \\ Email: sarkariais@upmc.edu.
}

\begin{abstract}
Robotic surgery continues to grow in thoracic surgery, and currently plays an evolving role in esophagectomy. Robotic assisted minimally invasive esophagectomy (RAMIE) has continued to expand, with many institutions adapting the technique. As the overall experience continues to grow, new data is emerging in its support. We present our approach to this operation.
\end{abstract}

Keywords: Esophagectomy; robotic; minimally invasive; esophageal cancer

Submitted Nov 25, 2019. Accepted for publication Jan 20, 2020.

doi: $10.21037 /$ jtd.2020.02.43

View this article at: http://dx.doi.org/10.21037/jtd.2020.02.43

\section{Introduction}

Esophagectomy was first described over one hundred years ago, and it continues to be an integral component in the treatment of esophageal cancer and occasionally for benign esophageal pathology (1-3). There are many approaches to esophagectomy. Regardless of the approach, historically, they all involved an open technique. Minimally invasive approaches to esophagectomy were first described in the 1990s, and over the last several decades, have become favored over open operations in many centers. Many studies have since described comparable outcomes between open approaches and total laparoscopic/thoracoscopic approaches $(4,5)$.

Over the last several decades, robotic minimally invasive techniques expanded to many operations. Robotic assisted minimally invasive esophagectomy (RAMIE) has emerged as an alternative approach to standard laparoscopic and thoracoscopic minimally invasive esophagectomy. The first descriptions of RAMIE were published in the early 2000s $(6,7)$. The RAMIE approach is increasing in popularity. A number of studies have demonstrated the safety of the procedure as well as oncologic outcomes similar to MIE and open operations (8-11). The robot offers several advantages to standard minimally invasive techniques which include superior instrument dexterity, magnified stereoscopic and central optics, and direct control of multiple working instruments allowing self-assistance. We describe our approach to a complete laparoscopic and thoracoscopic robotic-assisted Ivor Lewis esophagectomy using a fourarm robotic platform.

\section{Pre- and peri-operative planning}

All patients presenting with biopsy proven esophageal carcinoma undergo extensive clinical staging workup which includes endoscopic ultrasound, computed tomography, and fluorodeoxyglucose-18 positron emission tomography. Any comorbidities and overall fitness to undergo surgery are evaluated. Those with early-stage tumors confined to the mucosa are generally evaluated for possible therapy by endoscopic mucosal resection. Patients with T1b and T2 lesions and no evidence if lymphatic spread, are referred for surgery. Any patients with higher stage disease (T3 and/ or any $\mathrm{N}$ ) are referred for induction chemotherapy and radiation. After treatment, they are reevaluated for surgical resection.

Any patient that is a potential surgical candidate undergoes rigorous pre-operative testing to assess if they are fit enough to undergo esophageal resection. Typically patients are placed on a full liquid diet for several days prior to their operative date. The diet is reduced to clear liquids the day prior to operation.

On the day of surgery, patients are given prophylactic subcutaneous heparin in the holding area. The patient is intubated with a double-lumen endotracheal tube. 

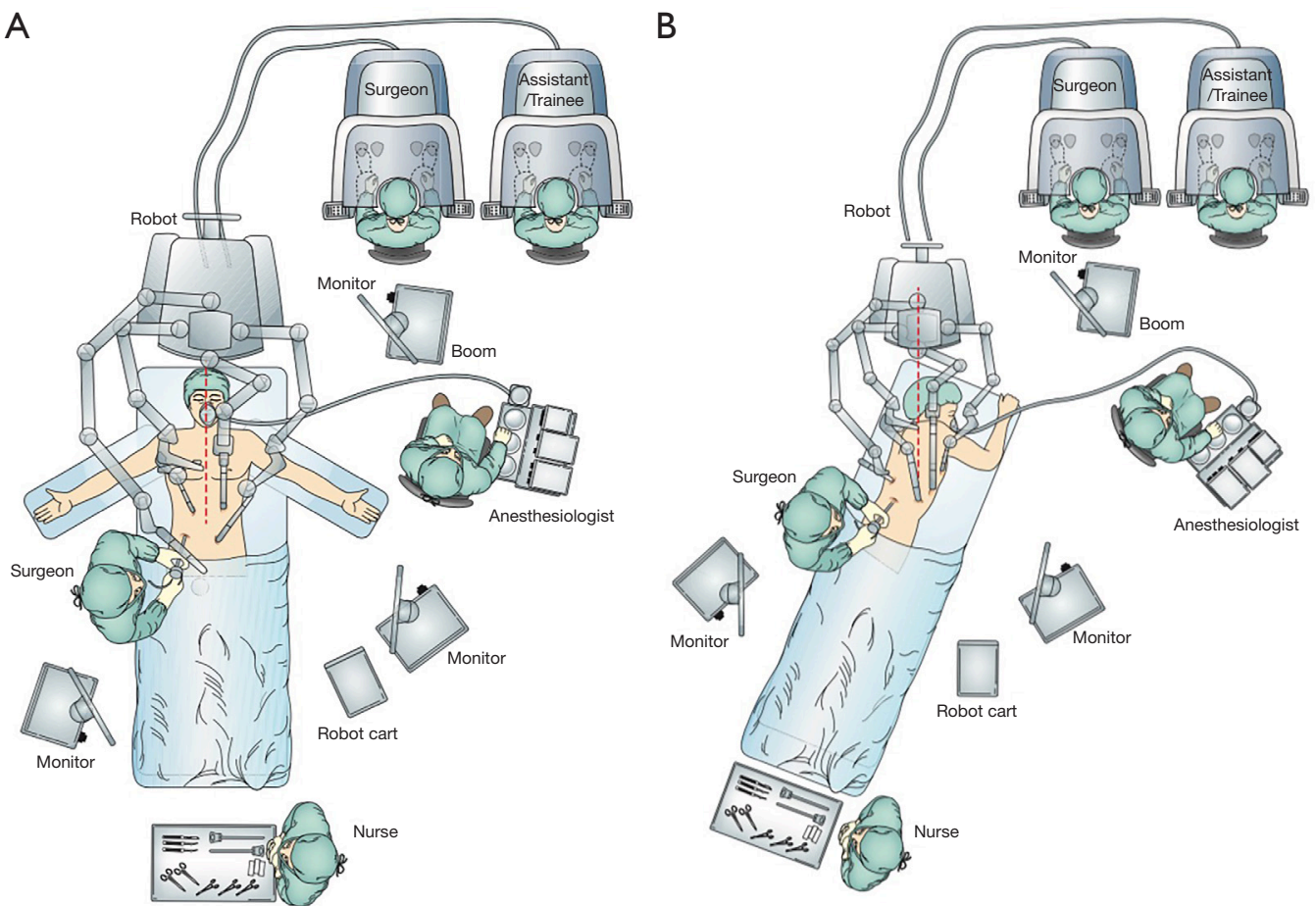

Figure 1 (A) Operating room setup during the abdominal phase of RAMIE; (B) during the thoracic phase, the patient is positioned in left lateral decubitus. [From (12) with permission].

Endoscopy is routinely performed to evaluate the tumor size, location, and extent onto the stomach.

The description generally applies to the DaVinci Xi platform (Intuitive Surgical, Sunnyvale, CA, USA). The robotic cart was traditionally brought in from the head of the patient, however, for newer platforms, the position of the cart is less relevant given the ability of the robotic gantry to rotate independent of the physical column structure. The patient is placed into reverse Trendelenburg position prior to attachment of the ports to the robotic arms. A footboard is in place to allow steep reverse Trendelenburg. The robotic cart is placed on the right on these cases by institutional convention and due to the operating room layout (Figure 1). Our institution uses two operating consoles which allows more direct surgical training. An assistant remains at the bedside. During the abdominal phase, the patient's positioned supine on the operative table with arms abducted $45^{\circ}$. For the thoracic phase, the patient is placed in standard left lateral decubitus position with the table flexed. The robotic cart is brought directly posterior from the patients right, and the gantry independently rotated to achieve the desired position of the arms in an oblique vector roughly facing the right shoulder.

\section{Port placement}

For the abdominal phase, it is important to mark the area of the hiatus, in which all instruments must be able to reach. An 8 -mm incision is marked just above the umbilicus which will serve as the camera port. It should be within $23 \mathrm{~cm}$ of the previously placed hiatal marker. A left lateral 8-mm incision just off the subcostal margin is marked for use for the robotic atraumatic grasper. A midclavicular 8-mm incision within $15-\mathrm{mm}$ from the hiatal mark is marked in the left mid abdomen. This port will be used for the dissecting energy device, which we prefer to use the ultrasonic shears. An additional $5-\mathrm{mm}$ port is marked in the right flank just beneath the subcostal margin for the liver retractor. A $12-\mathrm{mm}$ right midclavicular and midabdominal port is marked which will be initially for the bipolar atraumatic grasper but can accommodate robotic staplers. A 12-mm bedside assistant port is marked in the right lower quadrant between the umbilical and right midclavicular ports. This port can be upsized to $15-\mathrm{mm}$ to allow for larger stapler size, if hand-held staplers are used and larger stapler sizes are needed in the case of a thicker stomach. Port placement is outlined in Figure 2. 
A

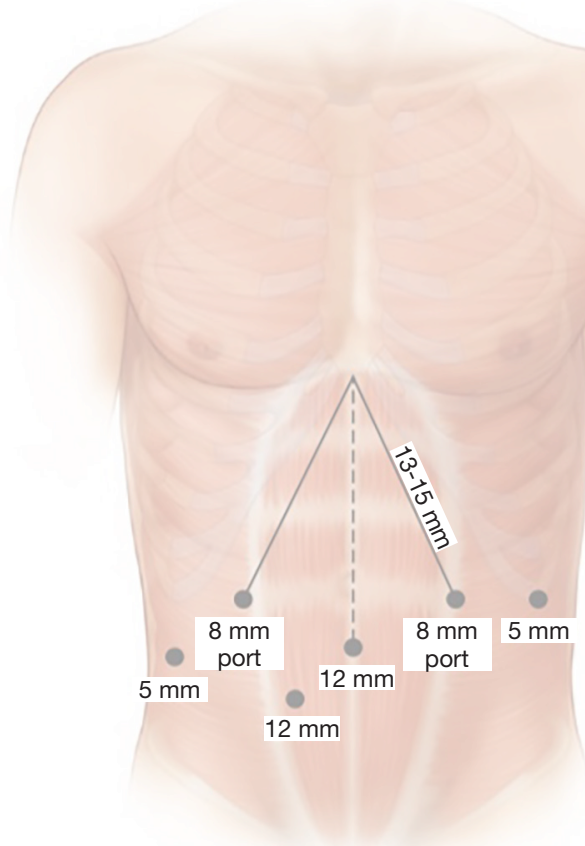

B

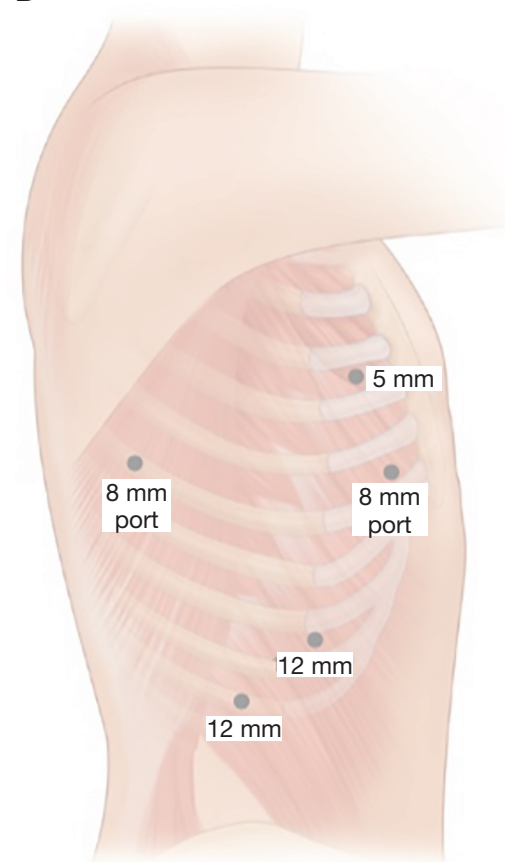

Figure 2 Size and location of post placement during the abdominal (A) and thoracic (B) phases of RAMIE. [From (12) with permission].

The patient is prepped and draped. The peritoneal cavity is accessed through a $12-\mathrm{mm}$ camera port using a direct Hassan trocar cutdown technique, or with a $5-\mathrm{mm}$ optical separator which can then be exchanged for an 8 -mm cannula. Once safe entry is obtained, pneumoperitoneum is obtained and set to a pressure of $15 \mathrm{mmHg}$. A standard laparoscope is used to evaluate for any metastatic disease. It should also be used for direct visualization of additional ports. After pneumoperitoneum is obtained, port locations may need to be adjusted to accommodate for any changes in distance in relation to the hiatus. A liver retractor is placed in the right lateral 5 -mm port and used to retract the left lateral lobe of the liver. Once all the ports are placed, the patient is placed in reverse Trendelenburg position. The robotic cart is then brought in as previously described and the robotic arms are docked to the ports. The instruments are then inserted under direct visualization.

For the thoracic phase, a 5-mm optical separator is used under direct visualization to enter the pleural space. The port is placed in the eight intercostal space in the midto posterior axillary line. Once safely in the intrapleural position, $\mathrm{CO}_{2}$ insufflation is instituted at a pressure of $8 \mathrm{mmHg}$ and the remain ports are then placed. Two 8-mm robotic ports are placed in the third and fifth intercostal spaces in the mid to posterior axillary line. An additional 8 -mm port is placed laterally in approximately the eighth or ninth interspace. A $12-\mathrm{mm}$ assistant port is placed at the diaphragmatic insertion between the camera port and the lateral most port. If robotic stapling is used, this is initially placed as a $12-\mathrm{mm}$ robotic stapler port and a $5-8$ mm cannula cap used to avoid loss of insufflation while in use by the bedside assist. The robot is then docked and the instruments and $30^{\circ}$ camera are inserted. The camera should be placed at a downward orientation. Figure 2 summarizes port placement.

\section{Surgical technique}

\section{Abdominal phase}

The dissection begins by incising the lesser sac. Care is taken to identify a potential replaced left hepatic artery. If one is identified, the extent of perfusion can be evaluated by occluding it and assessing the viability of the left lobe of the liver. If no compromise is identified, the artery can be sacrificed. Larger replaced arteries may need to be spared to avoid ischemia of the liver, but with care to maintain an adequate lymphadenectomy (13). Dissection then proceeds along the left gastric vascular pedicle, sweeping 


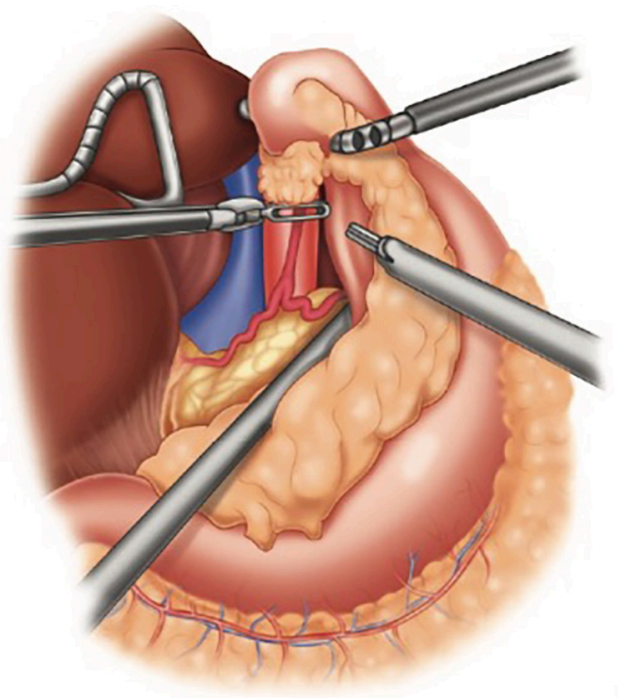

Figure 3 Dissection of the left gastric pedicle. The proximal left gastric artery is skeletonized and divided. All lymphatic tissue is swept toward the specimen side and removed en bloc. [From (12) with permission].

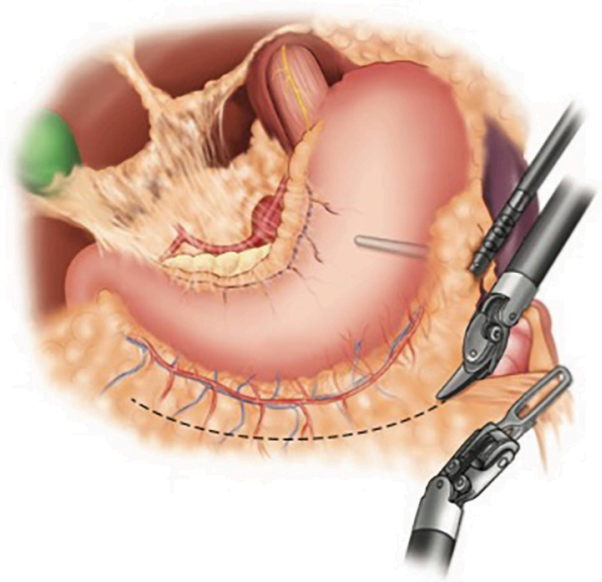

Figure 4 Gastric mobilization. The greater curvature of the stomach is mobilized by dividing the omentum and short gastric arteries. It is imperative to visualize and preserve the gastroepiploic arcade during this dissection. [From (12) with permission].

all associated lymphatic tissue toward the specimen side. The right crus is then dissected free. The dissection extends anteriorly and then the left crus is dissected in a similar fashion. If there is any concern for tumor involvement, portions of either or both crural can be removed. The esophagus is then circumferentially dissected along bilateral crural and pleural planes, anteriorly along the pericardium, and posteriorly along the aorta and vertebral column. During mediastinal dissection, the pleura may be violated. Although the majority of times it may not cause any acute physiologic changes, a low threshold should be had to place a tube thoracostomy in the event of any hemodynamic instability.

The retrogastric space is exposed by opening the lesser sac. The stomach is retracted anteriorly. Additional retraction can be proved by the bedside assistant. The stomach is then mobilized off the pancreas and splenic artery. All lymphatic tissue along the retroperitoneal plane is dissected out, up to the hiatus and then medially along the proximal common hepatic artery. Once completed, the left gastric vascular pedicle should be freely dissected (Figure 3). Any lymphatic tissue is swept toward the specimen. The celiac axis is then inspected for any gross disease or suspicious adenopathy that may alter the planned operation. Once freely dissected, the left gastric pedicle is divided with a robotic vascular load stapler. The stomach can be further retracted to expose any additional attachments to the left crural pillar.

After the gastroepiploic arcade is the identified along the greater curvature, the short gastric arteries are divided. Any remaining gastrosplenic attachments are divided and, if not already done, the left crus is completely mobilized. The omentum is then further divided and the stomach is completely mobilized to the level of the pylorus (Figure 4). It is imperative not to injury or jeopardize the gastroepiploic arcade during this dissection. In patients that underwent neoadjuvant radiation treatment, we prefer to harvest a pedicled omental flap. The flap is harvested in an area near the proximal portion of the eventual conduit. One or more dominant omental perforating arteries are identified and preserved for the flap. The flap will be later positioned around the anastomosis, between the conduit and airway. Indocyanine green injection may be given intravenously to visualize the vasculature with near-infrared imaging, which is standard on the $\mathrm{Xi}$ system. It is a useful adjunct to assess vascularity of the conduit, and better identify the vascular pedicle (14-16).

The antrum is retracted toward the left, exposing the pylorus. The pyloric muscle is identified by gently sweeping a handheld instrument along the antrum toward the duodenum to delineate the thickened muscle. Retraction stiches are then placed at the superior and inferior aspects of the pylorus. The pylorus is properly oriented by gentle traction on the retraction stitches. The pylorus is then 

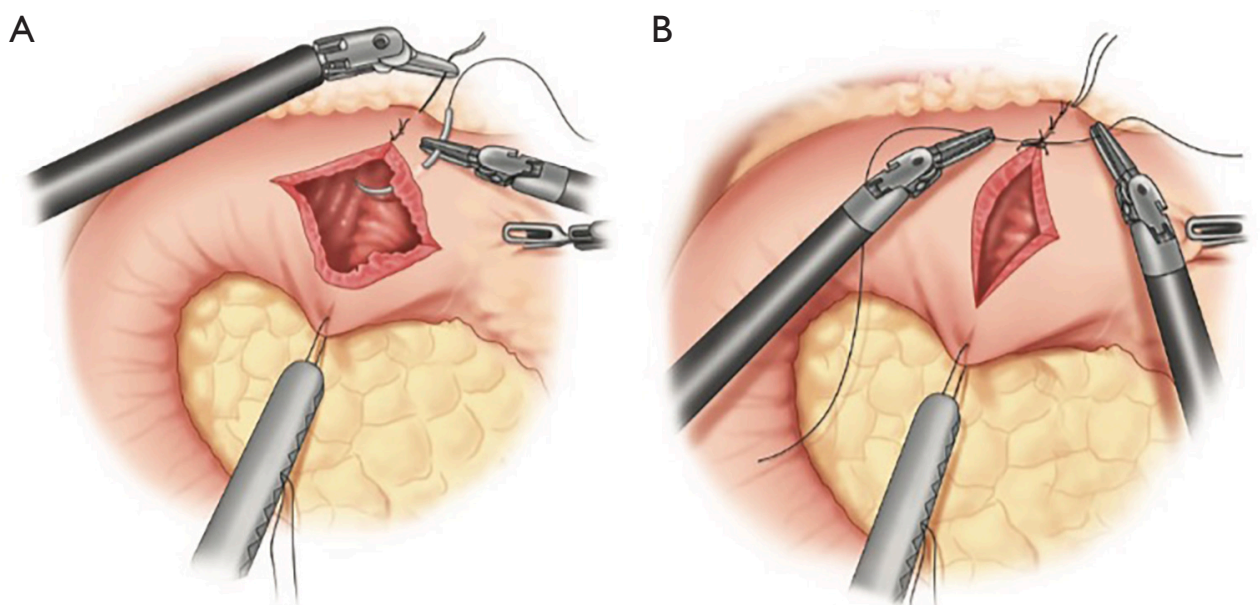

Figure 5 Pyloroplasty. A standard Heineke-Mikulicz technique is used to create the pyloroplasty. [From (12) with permission].

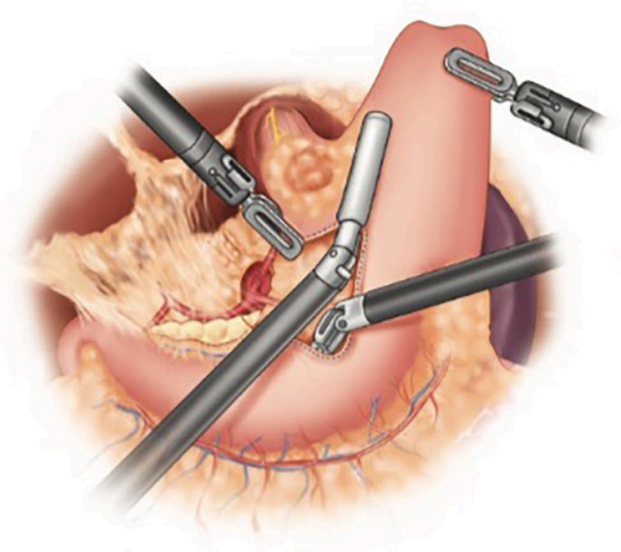

Figure 6 Creation of the gastric conduit. Multiple stapler fires are used to fashion a $3-3.5 \mathrm{~cm}$ wide conduit. [From (12) with permission].

opened full thickness in the longitudinal direction using an energy device, and then closed transversely. We prefer to use a 2-0 permanent braided suture (Figure 5). Typically, five to six total stiches are needed. A piece of omentum is tacked over the closure for further reinforcement.

When constructing the gastric conduit, the proximal most portion of the gastric fundus is retracted to the left upper quadrant using the left lateral robotic assistant arm. A robotic stapler is often used in the left robotic working hand through a $12-\mathrm{mm}$ port. Alternatively, a manual stapler can be brought through the bedside assistants $12-\mathrm{mm}$ trocar. The left lateral robotic arm can provide gentle downward retraction by posting along the neo-lesser curve as the staple line is formed. This serves to reduce redundancy of the tissues and straighten the stomach as the conduit is formed. The first stapler fire is used to divide the lesser curve vasculature at a point approximating the incisura. Then, using a series of staplers, a gastric tube is constructed maintaining a conduit width of approximately $3-4 \mathrm{~cm}$ (Figure 6). Each stapler should be parallel to the grater curvature of the stomach. Care is taken to maintain proper orientation of conduit. The short gastric line can be used as an aid to help prevent spiraling. We prefer a "no touch" technique during creation of the conduit, avoiding any grasping on any portion of usable conduit. Once completely divided, the conduit is secured to the specimen with heavy suture. It is later pulled through the hiatus during the thoracoscopic phase of the operation. If an omental flap was constructed, it is also secured to the tip of the conduit. We prefer to place a feeding jejunostomy on all esophagectomy patients. This is done with a standard laparoscopic approach. This then completes the abdominal phase of the operation.

\section{Thoracic phase}

The dissection begins by dividing the inferior ligament to the level of the inferior pulmonary vein. This dissection is carried down to the pericardium adjacent to the inferior vena cava. The esophageal hiatus is then mobilized to the contralateral pleura. With the lung retracted anteriormedially, the dissection proceeds by excising the mediastinal pleura superiorly. The airway is identified early. The subcarinal lymph nodes are carefully dissected (Figure 7). 

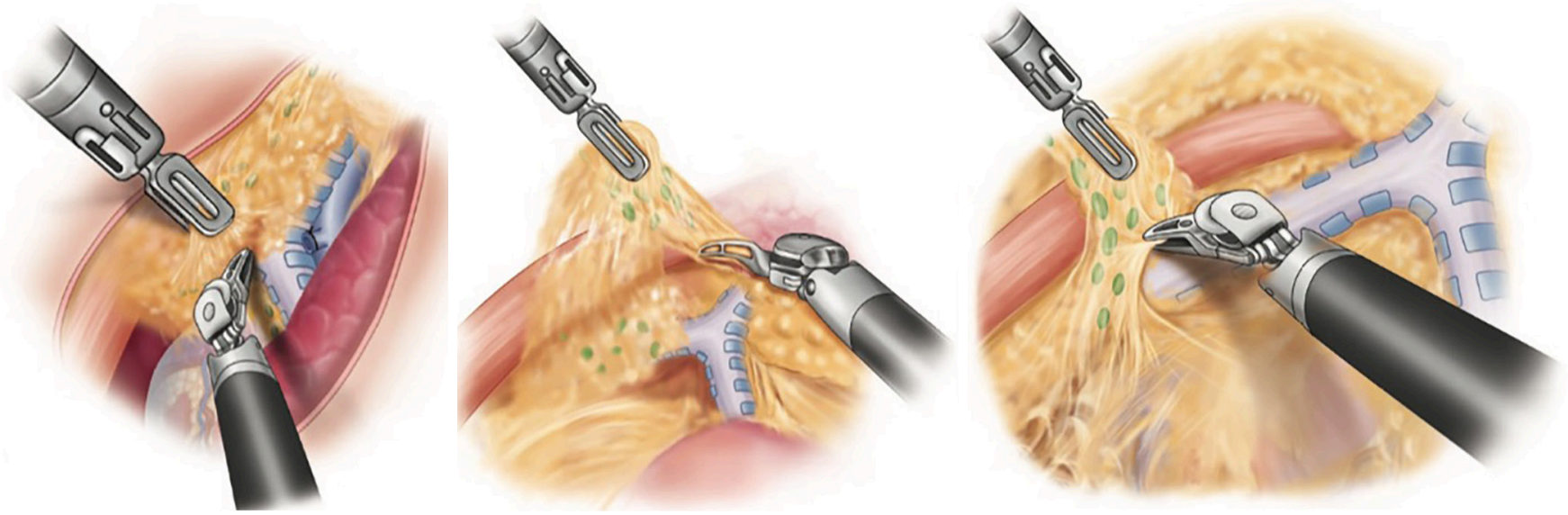

Figure 7 Subcarinal lymph node packet is removed en bloc. Extreme caution is used when using thermal energy to avoid injury the membranous trachea or left main stem bronchus. [From (12) with permission].

It is imperative to maintain a safe distance between the dissection plane and airway to avoid any thermal injury to the membranous airway. Although we prefer to use the ultrasonic shears for the majority of the dissection, bipolar energy may provide better thermal-energy containment for this portion of the procedure, especially in the early stages of the learning curve.

After complete dissection of the subcarinal nodal packet, the dissection continues superiorly to the azygous vein which is divided using a vascular stapler. The vagus nerve is identified and divided to prevent injury to the recurrent laryngeal nerve. Depending on the location of the tumor, the dissection may need to proceed up to the level of the thoracic inlet. The pleura posterior to the esophagus is then excised staying anterior to the thoracic duct. The dissection is carried down along the aorta to the hiatus. Surgical clips are used liberally for any lymphatics or sizable aortoesophageal arteries. The clips can be either robotically deployed or manually deployed by the bedside assistant. The thoracic duct is not routinely resected.

The conduit is then advanced into the chest, taking care to maintain proper orientation. The longitudinal staple line is used as a guide and should be facing laterally. The conduit is temporarily secured to the diaphragm to prevent retraction into the abdomen during the completion of the dissection. The specimen is retracted superiorly and laterally using the robotic assistant arm. The dissection is completed along the contralateral pleura and left mainstem bronchus. All nodal tissue is removed with the specimen. The esophagus is divided at the level above the azygous vein. The posterior "left-hand" port is removed and the incision is extended $4-\mathrm{cm}$. A wound retractor is placed and the specimen is removed and sent to pathology for evaluation of margins.

A running baseball purse-string suture using 0 permanent monofilament is placed along the circumferential opening (Figure 8). Each stitch should incorporate the muscular and mucosal layers, maintaining even depth of approximately $3-5 \mathrm{~mm}$. Once the stich is in place, an anvil is introduced into the esophagus and the purse-string is tied. An additional purse-string is then used to reinforce and secure the anvil.

The conduit is then advanced into the chest, again taking care to maintain proper orientation. The conduit is opened at the proximal most portion and held open. The end anastomotic stapler is introduced through the extended posterior incision and placed through the gastrotomy into the conduit. The conduit and stapler are positioned near the anvil. The stapler spike is deployed along the greater curve. The spike and anvil are married, and the stapler is fired (Figure 9). The stapler is then removed from the chest. The anastomotic "rings" are examined to confirm an adequate and complete anastomosis. A nasogastric tube is positioned in the conduit.

The redundant conduit containing the gastrotomy site is resected with the endo-gastrointestinal stapler. The distance between the anastomosis and gastrotomy closure should be approximately $2 \mathrm{~cm}$. If an omental pedicle flap was harvested, it is placed around the anastomosis and also used to interpose tissue between the conduit and the airway. 

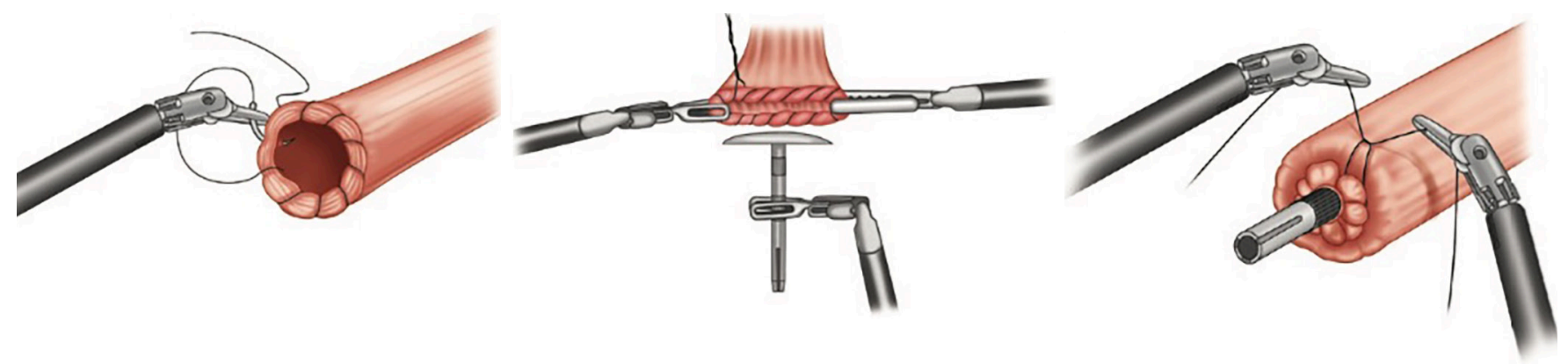

Figure 8 Placement of anvil. The anvil is secured into the proximal esophagus by first suturing a purse-string circumferentially prior to anvil insertion. An additional purse-string suture is added after the anvil is inserted. [From (12) with permission].
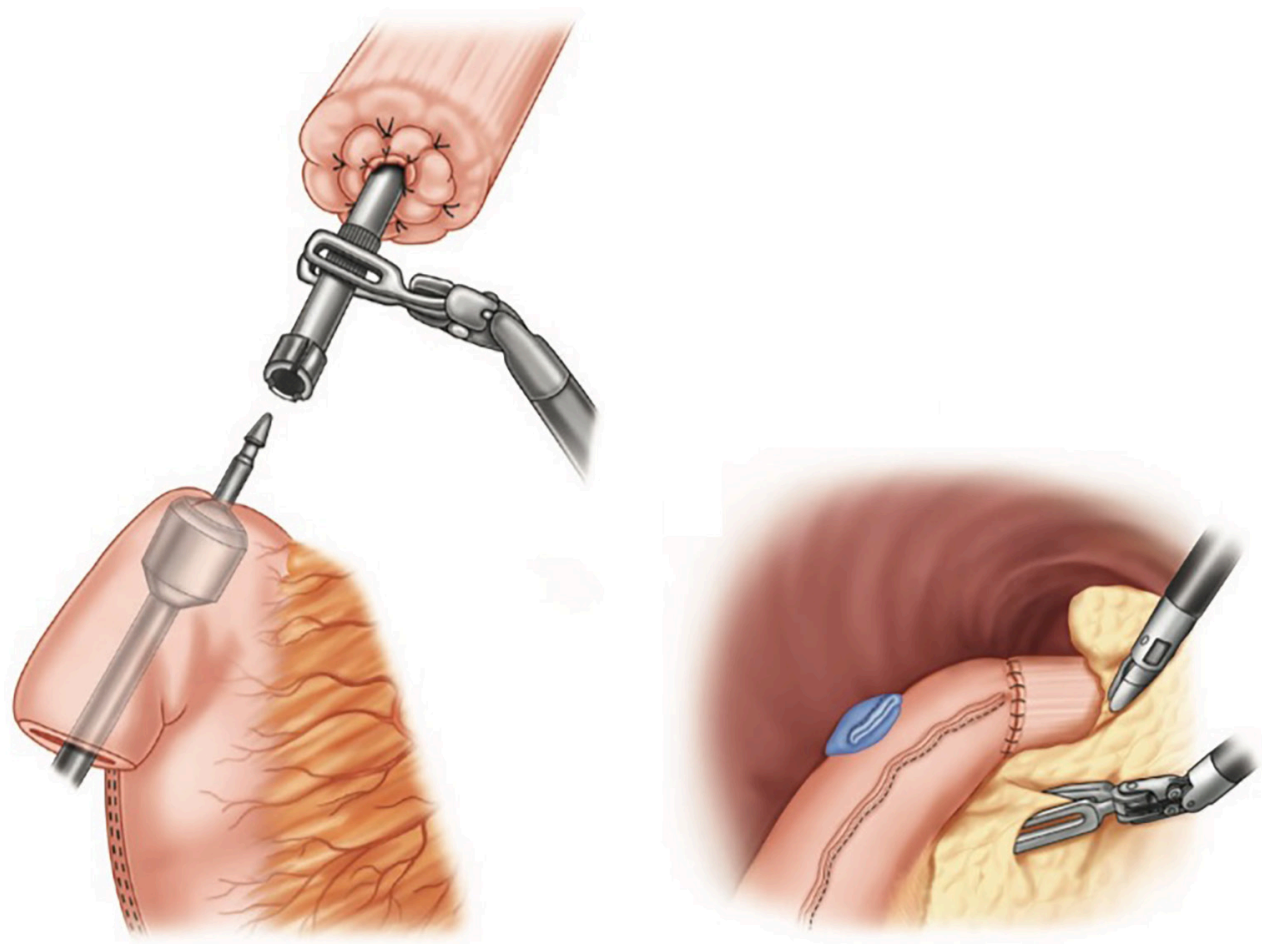

Figure 9 Anastomosis. The end-to-end anastomotic stapler is inserted into a gastrotomy site made in the distal aspect of the conduit. The stapler spike is then deployed and married to the anvil. Once proper orientation of the conduit is confirmed, the stapler is fired. [From (12) with permission].

After the chest is irrigated, a small drain (10-French flat Jackson-Pratt) is placed at the level of the anastomosis and connected to a suction free drainage bag. A single chest tube is placed.

\section{Postoperative care}

Prior to extubation, the double lumen endotracheal tube is exchanged for a single lumen and a toilet bronchoscopy is done. Patients remain in a monitored unit. Ambulation is started early in the recovery phase. Tube feeds are initiated 24-48 hours after surgery and slowly advanced. The nasogastric tube is typically removed on post-operative day 3 or sooner. An esophagram is performed between postoperative days 3-5. If no leak is identified, a quantitative step-wise liquid diet is started. Patient's length of hospital 
stays ranges around 6-8 days. The chest tube is removed in the early post-operative phase. Patients are discharged with the peri-anastomotic drain. The drain is removed in clinic on the first follow-up visit, and if tolerating a soft diet, the feeding jejunostomy can be removed.

\section{Discussion}

RAMIE has become increasing more utilized in the last decade. Although limited, the literature pertaining to RAMIE is expanding. Several institutions, including our own, have published early experiences. If performed in high volume centers, operative mortality is comparable to open esophagectomy $(\mathrm{OE})(10,11,17)$. Several centers have demonstrated that RAMIE can be done with minimal mortality risk when performed in experienced centers $(9,18)$. The major cause of morbidity following esophagectomy include pneumonia and respiratory failure. Although more data related to RAMIE is still needed, studies have shown that standard MIE results in decreased pulmonary related morbidity compared to open procedures (19).

One limitation of current prospective data available is the extent of "robotic assistance" in the esophagectomy, which may vary among surgeons and institutions. This inherently can create bias in comparisons. One of our authors has previously reported a prospective comparison of $\mathrm{OE}$ to a completely robotic approach (20). They reported a lower rate of pulmonary and infectious complications in RAMIE compared to OE. They also reported a lower anastomotic leak rate (3.1\% vs. 9.4\%), however, it was not statistically significant. Similarly, Meredith et al. (17) also noted a lower overall postoperative complication rate for RAMIE $v s$. OE, $23.6 \%$ vs. $30.5 \%$. No significant difference in anastomotic leaks rates was noted and overall perioperative mortality and length of stay were similar.

In a propensity matched study comparing RAMIE to OE using the National Cancer Database, Weksler et al. (21) found the 30-day mortality to be slightly higher in the RAMIE group (5.6\% vs. $2.7 \%, \mathrm{P}=0.061$ ), although it was not statically significant. The 90 -day mortality was similar. Espinoza-Mercato and colleagues (22) updated the analysis by Weksler's group with two additional years of data and found no difference in 30- and 90-day mortality. The significant learning curve associated with the procedure may have been possible explanation for this finding.

The recently published ROBOT trial represents a single center randomized controlled trial of RAMIE $v$ s. OE (23). To date, it is the only randomized controlled trial comparing
RAMIE to other traditional approaches. The study focused on surgery related postoperative complications. They reported overall lower surgical complication for RAMIE $v$ s. OE, $59 \%$ vs. $80 \%$. Both pulmonary complications $(32 \%$ vs. $58 \% ; \mathrm{P}=0.005)$ and cardiac complications (22\% vs. $47 \%$; $\mathrm{P}=0.006)$ were statistically less frequent in the RAMIE arm. Other complications, including anastomotic leak rate, overall mortality, and hospital stay, were not significantly different. The authors also reported improved functional recovery at 14 days, less mean overall postoperative pain scores, and improved quality of life measures in RAMIE $v s$. OE.

The Ivor Lewis RAMIE approach, as we described above, has shown to provide comparable oncologic outcomes. Multiple studies have demonstrated the feasibility of a complete resection, ranging from $90-100 \%(4,9,11,18,24)$. Several series have suggested an improved lymph node evaluation in the RAMIE $v s$. open $(17,20-22)$. Although the ROBOT trial focused primarily on perioperative outcomes, certain oncologic outcomes were evaluated. R0 resections were comparable between RAMIE and OE (93\% vs. 96\%, $\mathrm{P}=0.35)$ as well as median lymph nodes retrieved ( 27 vs. 25 nodes) (23). Although longer follow-up will be needed to draw any significant differences, at a median follow-up of 40 months, no significant difference in overall survival was noted between the two groups.

One major obstacle in the RAMIE is the learning curve associated with the procedure. This curve may vary based on the surgeon's comfort with robotic surgery as well as esophageal surgery. Hernandez and colleagues (25) attempted to define surgical proficiency for RAMIE. They concluded that near proficiency was obtained around 20 cases. In a study from Sarkaria et al. that included a cohort of 100 patients that underwent completely robotic RAMIE, the learning curve to achieve proficiency in terms of operative time and decreased complications was between 40-45 cases (18). The authors advocated several key principles for excellent outcomes including a dedicated and consistent operative team.

When first transitioning to RAMIE, it is important to focus on patient safety and outcomes. Success depends on many variables which include contributions from an entire team during the operation as well as the postoperative period. The surgeon and other members of the team should be prepared prior to first embarking on complex robotic esophageal surgery. Preparation includes simulation, observations of cases, cadaveric laboratory time, and appropriate proctoring. Appropriate preparation and 
experience will allow surgeons to excel and take advantage of the potential benefits of RAMIE as an additional tool in the growing armamentarium of minimally invasive approaches to these operations.

\section{Acknowledgments}

Funding: None.

\section{Footnote}

Provenance and Peer Review: This article was commissioned by the Guest Editor (Ghulam Abbas) for the series "Robotic Thoracic Surgery" published in Fournal of Thoracic Disease. The article has undergone external peer review.

Conflicts of Interest: All authors have completed the ICMJE uniform disclosure form (available at http://dx.doi. org/10.21037/jtd.2020.02.43). The series "Robotic Thoracic Surgery" was commissioned by the editorial office without any funding or sponsorship. Inderpal S. Sarkaria serves as an unpaid editorial board member of fournal of Thoracic Disease. The authors have no other conflicts of interest to declare.

Ethical Statement: The authors are accountable for all aspects of the work in ensuring that questions related to the accuracy or integrity of any part of the work are appropriately investigated and resolved.

Open Access Statement: This is an Open Access article distributed in accordance with the Creative Commons Attribution-NonCommercial-NoDerivs 4.0 International License (CC BY-NC-ND 4.0), which permits the noncommercial replication and distribution of the article with the strict proviso that no changes or edits are made and the original work is properly cited (including links to both the formal publication through the relevant DOI and the license). See: https://creativecommons.org/licenses/by-nc-nd/4.0/.

\section{References}

1. Giwa F, Salami A, Abioye AI. Hospital esophagectomy volume and postoperative length of stay: A systematic review and meta-analysis. Am J Surg 2018;215:155-62.

2. Li KK, Wang YJ, Liu XH, et al. The effect of postoperative complications on survival of patients after minimally invasive esophagectomy for esophageal cancer.
Surg Endosc 2017;31:3475-82.

3. Tapias LF, Mathisen DJ, Wright CD, et al. Outcomes With Open and Minimally Invasive Ivor Lewis Esophagectomy After Neoadjuvant Therapy. Ann Thorac Surg 2016;101:1097-103.

4. Luketich JD, Pennathur A, Awais O, et al. Outcomes after minimally invasive esophagectomy: review of over 1000 patients. Ann Surg 2012;256:95-103.

5. Luketich JD, Pennathur A, Franchetti Y, et al. Minimally invasive esophagectomy: results of a prospective phase II multicenter trial-the eastern cooperative oncology group (E2202) study. Ann Surg 2015;261:702-7.

6. Kernstine KH. The first series of completely robotic esophagectomies with three-field lymphadenectomy: initial experience. Surg Endosc 2008;22:2102.

7. van Hillegersberg R, Boone J, Draaisma WA, et al. First experience with robot-assisted thoracoscopic esophagolymphadenectomy for esophageal cancer. Surg Endosc 2006;20:1435-9.

8. Cerfolio RJ, Bryant AS, Hawn MT. Technical aspects and early results of robotic esophagectomy with chest anastomosis. J Thorac Cardiovasc Surg 2013;145:90-6.

9. Okusanya OT, Sarkaria IS, Hess NR, et al. Robotic assisted minimally invasive esophagectomy (RAMIE): the University of Pittsburgh Medical Center initial experience. Ann Cardiothorac Surg 2017;6:179-85.

10. Ruurda JP, van der Sluis PC, van der Horst S, et al. Robot-assisted minimally invasive esophagectomy for esophageal cancer: A systematic review. J Surg Oncol 2015;112:257-65.

11. Sarkaria IS, Rizk NP. Robotic-assisted minimally invasive esophagectomy: the Ivor Lewis approach. Thorac Surg Clin 2014;24:211-22, vii.

12. Safdie FM, Hess NR, Sarkaria IS. Robot-Assisted Minimally Invasive Esophagectomy (RAMIE): The Ivor Lewis Approach. In: Fong Y, Woo Y, Hyung WJ, et al. editor. The SAGES Atlas of Robotic Surgery. Berlin: Springer International Publishing AG, 2018.

13. Hess NR, Rizk NP, Luketich JD, et al. Preservation of replaced left hepatic artery during robotic-assisted minimally invasive esophagectomy: A case series. Int J Med Robot 2017. doi: 10.1002/rcs.1802.

14. Okusanya $\mathrm{O}, \mathrm{Lu} M$, Luketich JD, et al. Intraoperative near infrared fluorescence imaging for the assessment of the gastric conduit. J Thorac Dis 2019;11:S750-4.

15. Okusanya OT, Hess NR, Luketich JD, et al. Infrared intraoperative fluorescence imaging using indocyanine green in thoracic surgery. Eur J Cardiothorac Surg 
2018;53:512-8.

16. Sarkaria IS, Bains MS, Finley DJ, et al. Intraoperative near-infrared fluorescence imaging as an adjunct to robotic-assisted minimally invasive esophagectomy. Innovations (Phila) 2014;9:391-3.

17. Meredith KL, Maramara T, Blinn P, et al. Comparative Perioperative Outcomes by Esophagectomy Surgical Technique. J Gastrointest Surg 2020;24:1261-8.

18. Sarkaria IS, Rizk NP, Grosser R, et al. Attaining Proficiency in Robotic-Assisted Minimally Invasive Esophagectomy While Maximizing Safety During Procedure Development. Innovations (Phila) 2016;11:268-73.

19. Biere SS, van Berge Henegouwen MI, Maas KW, et al. Minimally invasive versus open oesophagectomy for patients with oesophageal cancer: a multicentre, open-label, randomised controlled trial. Lancet 2012;379:1887-92.

20. Sarkaria IS, Rizk NP, Goldman DA, et al. Early Quality of Life Outcomes After Robotic-Assisted Minimally Invasive and Open Esophagectomy. Ann Thorac Surg

Cite this article as: Witek TD, Brady JJ, Sarkaria IS. Technique of robotic esophagectomy. J Thorac Dis 2021;13(10):6195-6204. doi: 10.21037/jtd.2020.02.43
2019;108:920-8.

21. Weksler B, Sullivan JL. Survival After Esophagectomy: A Propensity-Matched Study of Different Surgical Approaches. Ann Thorac Surg 2017;104:1138-46.

22. Espinoza-Mercado F, Imai TA, Borgella JD, et al. Does the Approach Matter? Comparing Survival in Robotic, Minimally Invasive, and Open Esophagectomies. Ann Thorac Surg 2019;107:378-85.

23. van der Sluis PC, van der Horst S, May AM, et al. Robot-assisted Minimally Invasive Thoracolaparoscopic Esophagectomy Versus Open Transthoracic Esophagectomy for Resectable Esophageal Cancer: A Randomized Controlled Trial. Ann Surg 2019;269:621-30.

24. Hodari A, Park KU, Lace B, et al. Robot-Assisted Minimally Invasive Ivor Lewis Esophagectomy With Real-Time Perfusion Assessment. Ann Thorac Surg 2015;100:947-52.

25. Hernandez JM, Dimou F, Weber J, et al. Defining the learning curve for robotic-assisted esophagogastrectomy. J Gastrointest Surg 2013;17:1346-51. 